\title{
Introduction and expression of the bacterial PaeR7 methylase gene in mammalian cells
}

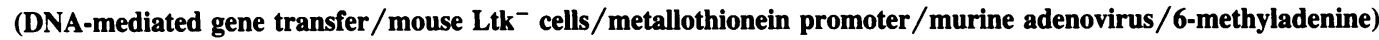

\author{
T. Jesse Kwoh, Deborah Y. Kwoh, Ann W. McCue, Geneva R. Davis, Denis Patrick, \\ AND ThOMAS R. GiNGERAS \\ La Jolla Biological Laboratories, San Diego, CA 92138-9216
}

Communicated by I. C. Gunsalus, July 11, 1986

\begin{abstract}
An approach is devised for studying the role of DNA methylation in eukaryotic gene expression. The approach is based on the expression of site-specific bacterial methylase genes in animal cells. A model system using the cloned $\mathrm{PaeR7}$ (an isoschizomer of $\mathrm{Xho} I$ ) methylase gene was constructed to test the feasibility of this approach. Expression plasmids for the $\mathrm{PaeR7}$ methylase gene were introduced into mouse Ltk $^{-}$cells by cotransfection with the cloned chicken thymidine kinase $(t k)$ gene. Several of the cell strains derived from $\mathrm{Tk}^{+}$colonies were found to express the $\mathrm{PaeR} 7$ gene as judged by four criteria: the cellular DNA of these strains showed increased resistance to cleavage by $X h o I$; these strains contained cellular proteins that comigrated with pure PaeR7 methylase protein, as visualized by immunoblotting; PaeR7 methylase activity was found in vitro in crude extracts of total cellular protein from these strains; and murine adenovirus genomes grown on cells expressing PaeR7 methylase showed resistance to cleavage to $P a e R 7$ endonuclease. The potential applications of this approach for the study of cellular and viral gene regulation, DNA repair, and restriction modification are discussed.
\end{abstract}

The mammalian genome contains the modified base 5methylcytosine, and this base occurs primarily at the dinucleotide CpG $(1,2)$. Several lines of evidence suggest that this DNA methylation is involved in the regulation of gene expression. First, a strong inverse correlation has been observed between the degree of methylation of specific genes and the activity of those genes (reviewed in ref. 3). Although exceptions have been found, most genes that are actively expressed are hypomethylated compared to inactive genes. Second, cloned mammalian genes that have been methylated in vitro are inactive when reintroduced into cells as long as the in vitro methylation pattern is maintained by cellular DNA methylases (4-7). Third, while the overall number of methylated bases within the mammalian genome is relatively invariant during development, the patterns of methylated sites within the DNA change in a tissue-specific manner (3).

Although the evidence strongly suggests that DNA methylation has a role in gene regulation, it is not yet known whether DNA methylation is involved in the primary events repressing gene expression, or whether DNA methylation is a secondary event that occurs on already inactivated genes. In addition, although it is assumed that the sites within or nearest the regulatory regions are the most important, it is also not yet known which methylation sites are critical for regulation of gene expression. Efforts to examine these questions have been hindered by the inability to manipulate methylation at specific sites within genes. In seeking to develop an approach for studying the role of DNA methyla-

The publication costs of this article were defrayed in part by page charge payment. This article must therefore be hereby marked "advertisement" in accordance with 18 U.S.C. $\$ 1734$ solely to indicate this fact. tion, we have sought to introduce and express site-specific bacterial methylase genes in mammalian cells. The study of the effect of such heterologous methylation on genes that are normally hypomethylated could offer insights into the biological significance of DNA methylation.

As a first step in demonstrating the feasibility of this approach, we used the PaeR7 methylase (an isoschizomer of Xho I), which methylates adenine in the sequence CTCGAG (8). In this report, we describe the introduction and expression of the PaeR7 methylase gene in mouse $\mathrm{Ltk}^{-}$cells.

\section{MATERIALS AND METHODS}

Purification of Plasmid and Bacteriophage DNA. Plasmids were introduced by $\mathrm{CaCl}$-mediated transformation (9) into and maintained in Escherichia coli strain MM294, K-12 F-, $\lambda^{-}, r_{K}^{-}, m_{K}^{+}$, endA, thi (10). Purified plasmid DNA was isolated from cleared lysates of chloramphenicol-treated cultures (11) by centrifugation through $\mathrm{CsCl}$ /ethidium bromide gradients. "Mini-preparations" of plasmid DNA were obtained by phenol lysis (12). Bacteriophage $\phi 80$ DNA was isolated from heat-induced cultures of CSH43, E. coli $\mathrm{K}-12$ tonA $\Delta($ lac $)$ thi $(\lambda c \mathrm{I} 857 \mathrm{St} 68 \mathrm{~h} 80)$, by the method described by Miller (13).

Construction of Expression Plasmids for Mammalian Cells. The construction scheme is outlined in Fig. 1. All restriction endonucleases and DNA-modifying enzymes were purchased from New England Biolabs and used according to the manufacturer's recommended conditions. Bgl II (TCCAGATCTGGA) and Sst I (TCCGAGCTCGGA) linkers and oligonucleotide probes used to screen the BAL-31 deletion library for $B g l$ II (TCCAGATCTGGAATGGGCATTTG, TCCAGATCTGGAATGGCCGCGA, TCCAGATCTGGATGGTCGATTT) and Sst I (TCCGAGCTCGGAATGGCATTG, TCCGAGCTCGGAATGGCCGCGA, TCCGAGCTCGGATGGTCGATTT) constructs were made using phosphoamidite chemistry in an Applied Biosystems model 380A DNA synthesizer.

Briefly, $\approx 30 \mu \mathrm{g}$ of pPAOM2.7 containing the PaeR7 methylase gene was cleaved with BamHI and partially digested with BAL-31 nuclease, and the fragment ends were repaired by the Klenow subfragment of DNA polymerase I (pol I). These digested fragments were ligated to $B g l$ II or Sst I linker oligonucleotides using T4 DNA ligase (14) and were used to transform $E$. coli. The resultant colonies were replicated onto nitrocellulose filters (15) and screened by hybridization to identify deletion mutants with $B g l$ II or Sst I linkers at positions in the PaeR7 methylase sequence immediately proximal to sequences that could be used as translational initiation sites. These sites had previously been identified as base pairs 592, 649, and 722 of the PaeR7 methylase gene by our characterization of the DNA sequence (16). Incrementally increasing temperature washes of the hybridization filters revealed the colonies possessing the highest affinity for each of the oligonucleotide probes. The 
position of the linker oligonucleotide relative to the $\mathrm{PaeR7}$ methylase gene sequence was confirmed by sequence analysis of M13mp18 or -mp19 clones containing each of the PaeR7 methylase deletions. Three plasmids were identified for further use: pPAO $\Delta 592$, pPAO $\Delta 649$, and pPAO $\Delta 721$.

The methylase gene present in each of these three plasmids was subcloned into a eukaryotic expression plasmid by insertion of an Sst I (for pPAO $\Delta 592$ ) or a $B g l$ II linker (for pPAO $\Delta 649$ and pPAO $\Delta 721$ ) into the unique $S a l$ I site of each plasmid. DNA fragments containing the PaeR7 methylase gene were isolated by first cleaving pPAO $\Delta 592$ with Sst I and pPAO $\Delta 649$ and pPAO $\Delta 721$ with $B g l$ II. The desired DNA fragments were isolated from a $1.0 \%$ agarose gel (17). Eukaryotic expression plasmid pMMT341 (obtained from M. F. Law) and its derivative pMMT341S were digested with $B g l$ II and Sst I, respectively, and corresponding fragments from pPAO $\Delta 592$, pPAO $\Delta 649$, and pPAO $\Delta 721$ were ligated onto these sites. Plasmids containing the $\mathrm{PaeR} 7$ methylase gene in the correct orientation with respect to the mouse metallothionein promoter were designated -1 ; the opposite orientation was designated -2 .

Transfection of Mouse Cells and Cell DNA and Protein Preparation. Mouse $\mathrm{Ltk}^{-}$cells were grown at $37^{\circ} \mathrm{C}$ in $5 \%$ $\mathrm{CO}_{2} / 95 \%$ air in Dulbecco's modified Eagle's medium, supplemented with $10 \%$ calf serum/penicillin (100 units $/ \mathrm{ml}) /$ streptomycin $(100 \mu \mathrm{g} / \mathrm{ml})$ (GIBCO). Cotransfection of mouse Ltk $^{-}$cells using the PaeR7 methylase gene constructions, the chicken thymidine kinase gene (pCHTK5) (10), and bacteriophage $\phi 80$ DNA was performed as described (18). After 2 weeks in selective medium containing hypoxanthine $(15 \mu \mathrm{g} / \mathrm{ml})$, aminopterin $(1 \mu \mathrm{g} / \mathrm{ml})$, and thymidine $(5 \mu \mathrm{g} / \mathrm{ml})$, individual colonies were isolated and grown to mass culture. Total cellular DNA was prepared from $2 \times 10^{8}$ cells as described (18). Total cellular protein extracts were prepared from $4 \times 10^{7}$ actively growing cells. These cells were resuspended in $1.0 \mathrm{ml}$ of $100 \mathrm{mM}$ Tris $\mathrm{HCl}, \mathrm{pH} 7.4 / 100 \mathrm{mM}$ 2-mercaptoethanol/5 mM EDTA/1 mM phenylmethylsulfonyl fluoride/chymostatin $(2 \mathrm{mg} / \mathrm{ml}) / 5 \mathrm{mM} p$-aminobenzamidine, and lysed by brief sonication.

In Vitro PaeR7 Methylase Assay. Two reactions were performed for each cell line. Each reaction mixture contained $20 \mu \mathrm{l}$ of total protein extract with $2 \mu \mathrm{g}$ of pX164 DNA in a final vol of $150 \mu$ l containing $50 \mathrm{mM}$ Tris $\mathrm{HCl}(\mathrm{pH} 7.4), 10 \mathrm{mM}$ EDTA, and $10 \mathrm{mM}$ 2-mercaptoethanol. Plasmid pX164 is pBR322 containing one $X$ ho I linker inserted at position 164 (10). One of the two reaction mixtures contained $0.1 \mathrm{mM}$ $S$-adenosyl-L-methionine (Sigma). After incubation at $37^{\circ} \mathrm{C}$ for $30 \mathrm{~min}$, the reactions were phenol-extracted and ethanolprecipitated. The precipitated pX164 DNA was redissolved in $50 \mu \mathrm{l}$ of $\mathrm{PaeR} 7$ buffer $(6 \mathrm{mM}$ Tris $\cdot \mathrm{HCl}, \mathrm{pH} 7.4 / 6 \mathrm{mM}$ $\mathrm{MgCl}_{2} / 6 \mathrm{mM}$ 2-mercaptoethanol/100 $\mu \mathrm{g}$ of bovine serum albumin per ml). One-half of the DNA was digested with $P a e R 7$ or $X h o$ I endonuclease for $2 \mathrm{hr}$ at $37^{\circ} \mathrm{C}$. The digested and undigested pX164 DNA was resolved by agarose gel electrophoresis and then transferred to nitrocellulose filters using Southern hybridization procedures (19). These filters were probed with nick-translated pBR322.

Preparation of Anti-PaeR7 Methylase Antiserum and Immunoblotting. A rabbit was immunized with three multisite subcutaneous injections of purified PaeR7 methylase, which was isolated as described (16). The injections were 8 or 9 days apart, and $25 \mu \mathrm{g}$ of protein was administered at each injection. Freund's complete adjuvant (Calbiochem-Behring) was used for the first injection, and Freund's incomplete adjuvant was used for the two subsequent injections. Antiserum was prepared from blood taken 11 and 21 days after the last injection.

For each cellular protein extract, $8 \mu \mathrm{l}$ of extract was fractionated by $\mathrm{NaDodSO}_{4} / 8 \%$ polyacrylamide gel electrophoresis with a $5 \%$ stacking gel. Proteins were transferred to nitrocellulose by electroblotting (20) for immunoblot hybridization analysis. Nitrocellulose filters were washed for $1 \mathrm{hr}$ at room temperature in $1 \times$ buffer $B$ [0.25\% gelatin (Bio$\mathrm{Rad}) / 0.05 \%$ Tween 20 (Bio-Rad)/0.02\% sodium azide/1X phosphate-buffered saline], and underwent reaction with a 1:200 dilution (in $1 \times$ buffer B) of methylase antisera for $3 \mathrm{hr}$ at $4^{\circ} \mathrm{C}$ with shaking. After washing three times ( 20 min each) at room temperature in $1 \times$ buffer $B$, the filters were placed in a $50-\mathrm{ml}$ solution containing $1 \times$ buffer $\mathrm{B}$ and $0.5 \mu \mathrm{Ci}$ of ${ }^{125} \mathrm{I}$-labeled protein A per lane $(1 \mathrm{Ci}=37 \mathrm{GBq})(\mathrm{New}$ England Nuclear) at room temperature for $1 \mathrm{hr}$ with shaking. The filters were washed four times ( $20 \mathrm{~min}$ each) in $1 \times$ buffer $B$, blotted dry, and autoradiographed.

Growth and Purification of Murine Adenovirus. Mouse adenovirus strain FL (obtained from K. R. Spindler) was grown and purified according to Larsen and Nathans (21), except that mouse $\mathrm{Ltk}^{-}$or $\mathrm{TK}^{+}$transfectants were used as host cells. Mouse adenovirus DNA was prepared from purified viral particles as described (21).

\section{RESULTS}

Genomic DNA Resistance to Xho I Cleavage. The construction of mammalian expression plasmids for the PaeR7 methylase gene is outlined in Fig. 1. Three plasmids were made: one to the putative natural initiation codon (GTG) and two to upstream in-frame ATG codons. These plasmids (500 ng) were introduced into mouse $\mathrm{Ltk}^{-}$cells by cotransfection with $50 \mathrm{ng}$ of pCHTK 5 [chicken thymidine kinase $(t k)$ gene] and $500 \mathrm{ng}$ of $\phi 80$ phage DNA per plate. TK ${ }^{+}$colonies were cloned and grown to mass culture. The phage DNA was included to aid in the examination of the isolated cells, since Xho I (an isoschizomer of PaeR7) restriction sites are relatively infrequent in mouse chromosomal DNA but are plentiful in the $\phi 80$ phage genome. We isolated 5-10 colonies from each cotransfection type. All the resulting cell strains contained DNA hybridizable to the methylase expression plasmids (data not shown). Southern blot hybridization of the $\phi 80$ phage sequences within the cells after cleavage with $X$ ho I, BamHI, and Xho I plus BamHI showed that the DNA from some of these cells was resistant to $X$ ho I cleavage. Fig. 2 shows the Southern blot hybridization profiles for three cell strains. The parental cell line, $\mathrm{Ltk}^{-}$, does not contain pCHTK5, $\phi 80$ DNA, or methylase expression plasmids and shows that the $\phi 80$ sequences added to cellular genomic DNA are sensitive to Xho I cleavage in the absence of the methylase activity. This sensitivity is characterized by the appearance of several bands in the Xho I-digested DNA, compared to the undigested DNA, and by the shift in the sizes of several bands in a BamHI digest as compared to the BamHI plus Xho I digest. In contrast, cell strain A24b1 (which received plasmid pPAO592MT-1) and strain B6b2 (plasmid pPAO649MT-1) show none of the Xho I-generated $\phi 80$ fragments identified in the $\mathrm{Ltk}^{-}$line and thus appear to be resistant to $X$ ho I cleavage. In addition, the patterns for $B a m \mathrm{HI}$ alone and the BamHI plus Xho I double digests for these cell strains appear to be identical. The resistance to $X$ ho I cleavage suggests that the $P a e R 7$ methylase is expressed in these strains. The ability of BamHI to digest the chromosomal DNA suggests that the resistance to Xho I is not part of a general artifact caused by DNA purification methods. Other cell lines, transfected with $\phi 80$ and pCHTK 5 but no methylase gene, or plasmids with incorrect orientation for the methylase gene show no protection of $\phi 80$ sequences from $X$ ho I digestion (data not shown).

A total of four cell strains that exhibited strong resistance to Xho I cleavage were selected for further study. Of these, one line contained pPAO592MT-1, and three contained pPA0649MT-1. Of 10 isolated $\mathrm{TK}^{+}$cell lines transfected with pPA0721MT-1, none exhibited resistance to $X$ ho I cleavage, 


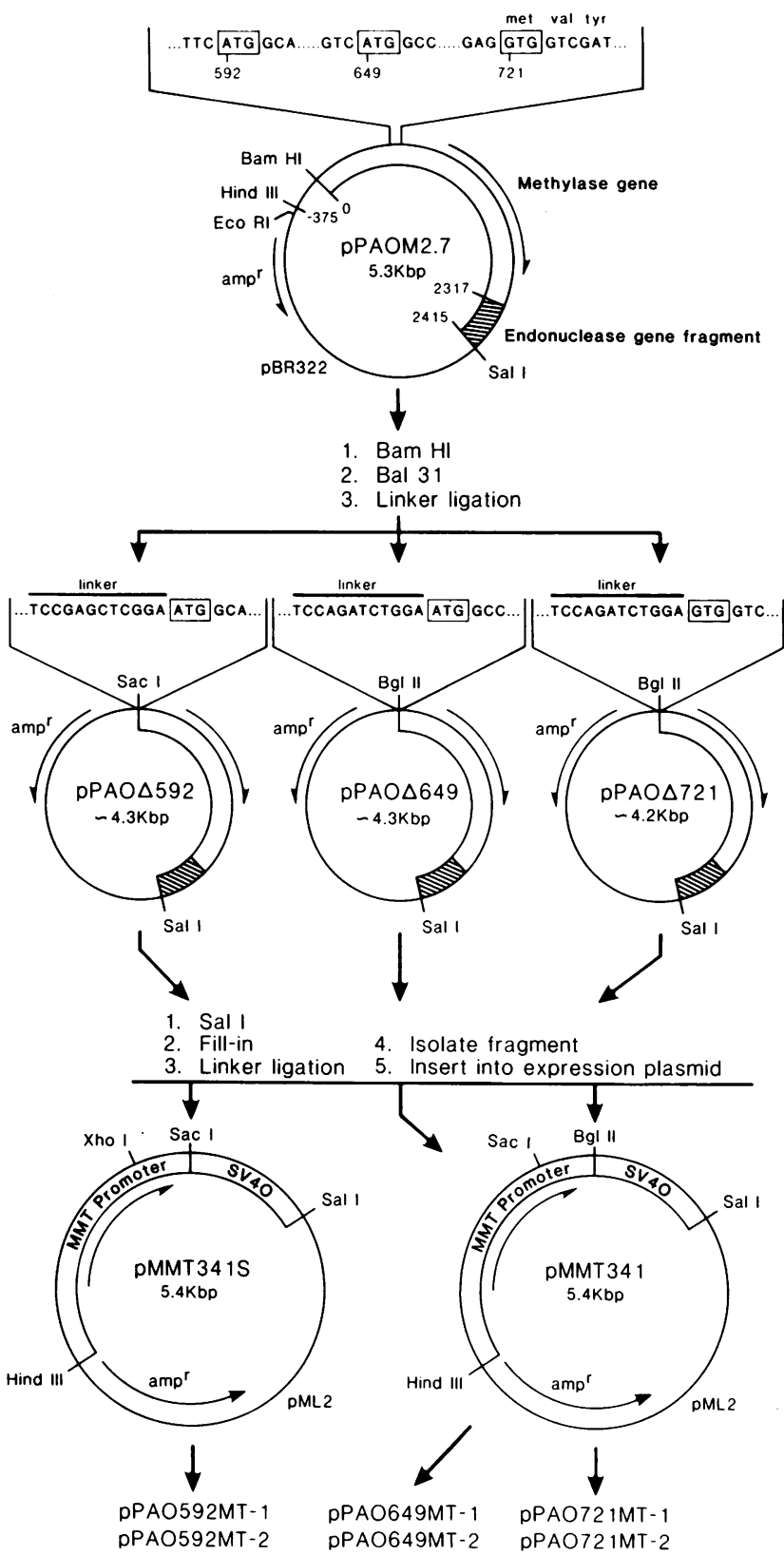

Fig. 1. Construction of PaeR7 expression vectors. The PaeR7 methylase gene was deleted from the extraneous upstream sequence and inserted into plasmids containing the mouse metallothionein promoter and simian virus $\mathbf{4 0}$ small tumor antigen RNA processing sequences.

suggesting that this construct does not express the methylase. This lack of expression is not surprising, since only a GTG codon could be used as a translational initiation site.

Detection of Methylase Protein by Immunoblot Hybridization. Three additional strains, which showed no protection from Xho I cleavage, were selected at random as controls. One of the control strains, A4b3, never received any methylase expression plasmid. The other two, A16b2 and A26c3, received pPAO721MT-1 but did not exhibit protection from $X h o$ I cleavage. These cell strains, along with $\mathrm{Ltk}^{-}$, were propagated, and extracts of total cell protein were fractionated by $\mathrm{NaDodSO}_{4} /$ polyacrylamide gel electrophoresis, transferred to nitrocellulose, and probed with antiM.PaeR7 antiserum. Fig. 3 shows the results of one such experiment. In the extracts from the four cell strains whose DNA was Xho I resistant, proteins that comigrate with at least one of the two purified methylase proteins were observed. The PaeR7 methylase enzyme is present in two forms
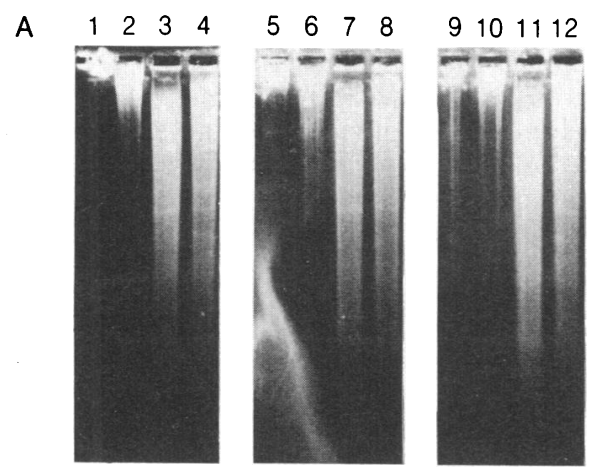

B
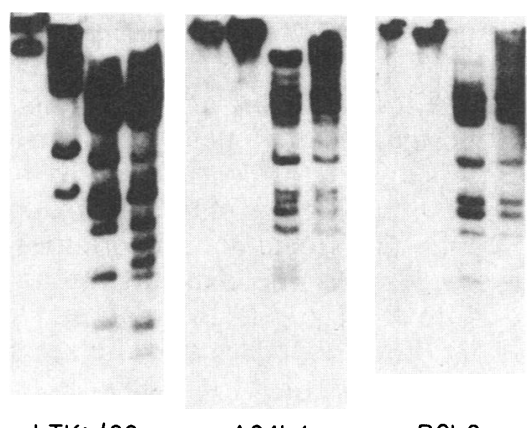

$L T K+\phi 80$

A24b 1

B6b2

Fig. 2. Restriction enzyme digestion and Southern blot hybridization of total cellular DNA. Total cellular DNA for strains Ltk DNA plus $\phi 80$ DNA (lanes 1-4), A24b1 (lanes 5-8), and B6b2 (lanes 9-12) was untreated (lanes 1, 5, and 9) or digested with Xho I alone (lanes 2, 6, and 10), BamHI alone (lanes 3, 7, and 11), or both Xho I and BamHI (lanes 4, 8, and 12). After agarose gel electrophoresis $(A)$, digested DNA was transferred to nitrocellulose filters. Bacteriophage $\phi 80$ DNA was ${ }^{32} \mathrm{P}$-labeled by nick-translation and used as the hybridization probe for the DNA on the filter $(B)$.

as isolated from $E$. coli cell lines expressing this gene. It is believed that these forms result from multiple translational initiation sites (16). The eukaryotic expression plasmids used in this study employ one GTG (pPAO721MT-1) and two ATG (pPAO592MT-1 and pPAO649MT-1) potential translational initiation sites. The pPA0592MT-1-derived cell line A24b1 exhibits two polypeptides that comigrate with the two purified methylase bands, whereas the pPA0649MT-1-derived strains B6b2, B6c1, and B11b1, exhibit a single band that

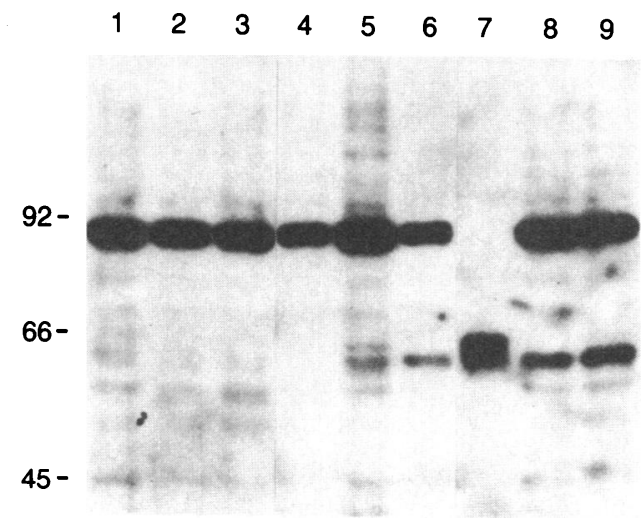

Fig. 3. Immunoblotting of total cellular protein. After fractionation of total cellular protein by $\mathrm{NaDodSO}_{4} /$ polyacrylamide gel electrophoresis, the proteins were transferred to nitrocellulose filters by electroblotting. The filters were probed with rabbit anti-PaeR7 methylase antiserum, and bound antibodies were detected by using ${ }^{125}$ I-labeled protein A. Lanes: 1, Ltk; 2, A4b3; 3, A16b2; 4, A26c3; 5, A24b1; 6, B6b2; 7, pure PaeR7 methylase; 8, B6c1; and 9, B11b1. The position of protein size markers $(\mathrm{kDa})$ is shown on the left. 
comigrates with the smaller purified methylase protein. Neither of the two bands that comigrate with the purified methylase was detected in the control cell lines.

In Vitro Methylase Activity. The total cell protein extracts from these cell lines were assayed for methylase activity in vitro. Fig. 4 shows that crude extracts of strains A24b1 and B6b2 are able to partially modify pX164 DNA, a plasmid that contains an Xho I site, and thereby preserve the supercoiled form of $\mathrm{pX164}$, whereas extracts from A4b3 and Ltk cannot. Strains B6c1 and B11b1 also contained PaeR7 methylase activity (data not shown). The quantities of methylase in cell lines A24b1 and B6b2 observed by immunoblot hybridization experiments (Fig. 3) are similar to the quantity of purified methylase used as a reference. However, this quantity of purified methylase is sufficient to totally protect the pX164 plasmid (data not shown), whereas only partial protection is observed from extracts A24b1 and B6b2. Such partial protection may be the result of methylase instability in crude extracts. Because no supercoiled DNA is observable for pX164 treated with extracts from strains $A 4 \mathrm{~b} 3$ and Ltk or from A16b2 and A26c3 (data not shown), these cell lines do not exhibit any in vitro PaeR7 methylase activity. On the other hand, both B6c1 and B11b1 (data not shown), along with A24b1 and B6b2, exhibit methylase activity.

Methylation of Adenovirus Grown on Methylase-Positive Cell Lines. Murine adenovirus was used to infect Ltk, B6b2, and $\mathrm{A} 4 \mathrm{~b} 3$ cell lines at a multiplicity of infection of $0.1-0.3$. After 10 days of growth, the virus was isolated from these cells, and DNA was purified from the viral particles. This DNA was cleaved with PaeR7 and BamHI to determine whether the viral genome had been methylated. Fig. 5 reveals that adenovirus grown on cell lines Ltk and A4b3 is sensitive to cleavage by the PaeR7 endonuclease. However, virus grown on $\mathrm{B} 6 \mathrm{~b} 2$ is largely resistant to $\mathrm{PaeR} 7$ cleavage, indicating that methylation of the murine adenovirus genome has occurred. In subsequent experiments, viral particles containing methylated genomes were used to infect the B6b2 cell line. Adenovirus DNA recovered from this second passage through a $P a e R 7$ methylase-expressing host showed identical digestion patterns, as displayed in Fig. 5 (lanes 5 and 6), after cleavage with $P a e R 7$ and BamHI, respectively (data not shown). Thus, the levels of methylation of the murine adenovirus genome remained constant after two successive passages through a methylase-expressing host.

\section{DISCUSSION}

One roadblock to understanding the level at which DNA methylation in eukaryotic cells operates to regulate gene

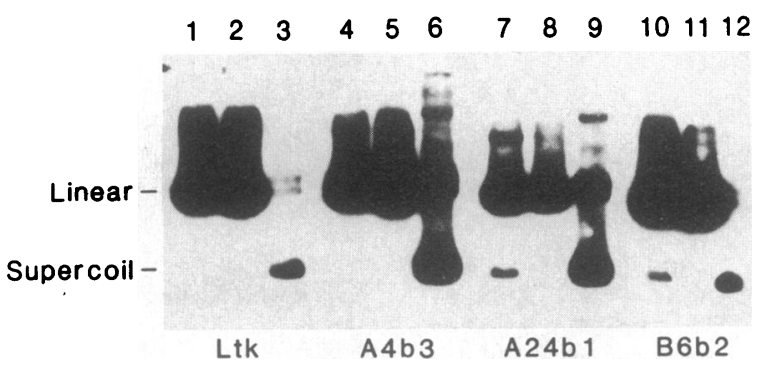

FIG. 4. In vitro methylase assay of crude cell extracts. Plasmid pX164 was incubated with total cellular protein extract from strains Ltk (lanes 1-3), A4b3 (lanes 4-6), A24b1 (lanes 7-9), and B6b2 (lanes 10-12), either with (lanes $1,4,7$, and 10) or without (lanes $2,3,5,6$, $8,9,11$, and 12) $S$-adenosyl-L-methionine. After phenol extraction and ethanol precipitation, the DNA was either digested (lanes 1, 2, $4,5,7,8,10$, and 11) or not digested (lanes $3,6,9$, and 12) with PaeR7 endonuclease and fractionated by agarose gel electrophoresis. The DNA was transferred to nitrocellulose and hybridized using ${ }^{32} \mathrm{P}$ labeled nick-translated pBR322 as the probe. Cleavage of pX164 by PaeR7 converts the supercoiled plasmid to the linear form.

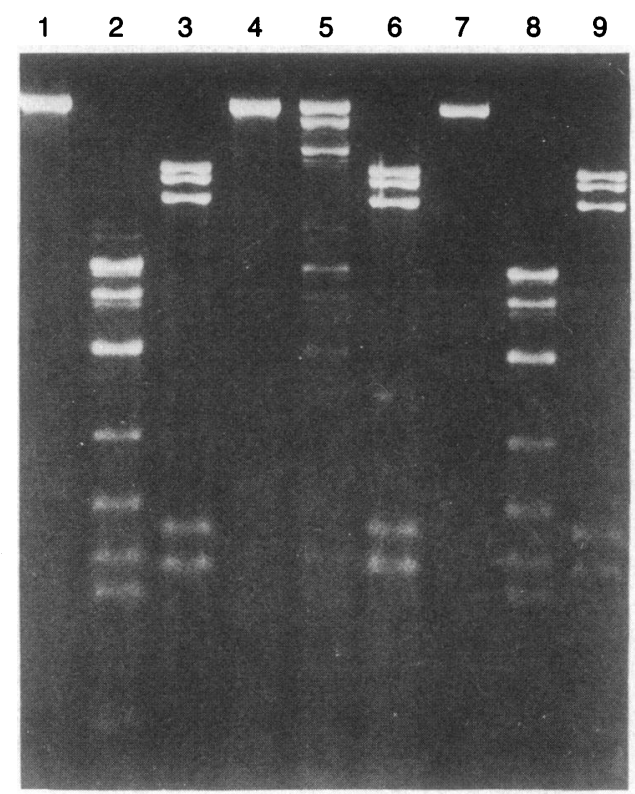

FIG. 5. Restriction enzyme digests of murine adenovirus grown on methylating and nonmethylating cell lines. MAV-FL DNA (300 $\mathrm{ng}$ ), prepared from virus grown on mouse $\mathrm{Ltk}^{-}$(lanes 1-3), B6b2 (lanes 4-6), and A4b3 (lanes 7-9) was either undigested (lanes 1, 4, and 7) or digested with PaeR7 (lanes 2, 5, and 8) or BamHI (lanes 3, 6 , and 9), and then fractionated on a $1 \%$ agarose gel. The DNA was visualized with ethidium bromide and UV lights.

expression is an inability to manipulate the methylation of specific sites within specific genes. Two types of experiments have been reported to address this problem. In one type of study, the methylation pattern of specific genes has been manipulated by in vitro modification of the genes, followed by reintroduction into animal cells (4-7). This in vitro methylation has been accomplished by use of bacterial site-specific methylases or by the use of 5-methyldeoxycytidylate triphosphate during DNA synthesis. While such studies attempt to examine changes in the methylation pattern of specific genes, they suffer the disadvantage that the imposed methylation pattern may or may not be maintained by the host cell's own DNA methylase. Although many in vitro-methylated $\mathrm{CpG}$ sites are maintained in mammalian cells, some are not, and a slow loss of methylation is observed over time at many in vitromodified sites. With regard to this latter point, many sites modified by the incorporation of 5-methylcytosine cannot be monitored for methylation since they occur within sequences not cleaved by any restriction endonuclease.

The second type of study in which methylation patterns of specific genes are manipulated involves the treatment of mammalian cells with an inhibitor of DNA methylation (22-24), 5-azacytidine. Cells grown in the presence of this agent lose their ability to carry out DNA methylation and consequently exhibit an extremely hypomethylated genome. However, because of side effects of this drug [chromatin breakage (25) and exchange (26)], phenotypic results observed with this agent are difficult to interpret unambiguously. In addition, alterations in the methylation pattern of large segments of the genome accompany the loss of methylation of a specific target gene.

We have taken another approach to studying the relationship of DNA methylation and gene expression. This approach involves the expression of site-specific bacterial methylase in mammalian cells. In one way, such an approach is similar to the in vitro methylation studies, except that maintenance of methylation is now dependent on the in vivo expression of the bacterial methylase. Another advantage of this new approach is that all of the methylated sites generated by the bacterial 
gene can easily be assayed with the cognate restriction enzyme. Previous studies examining tissue-specific changes of a methylation pattern suffered the disadvantage that not all sites could be assayed.

One difficulty with our new approach is that the introduction of bacterial methylases, especially ones that synthesize 5-methyl CpG, may be detrimental to the growth of mammalian cells. For this reason, we initially used the PaeR7 methylase gene to test the feasibility of our approach. The PaeR7 enzyme methylates adenines in the sequence CTCGAG (8). Previous reports on the introduction of the $E$. coli dam gene into yeasts suggest that high adenine methylation of the yeast genome has no effect on cell metabolism (27, 28). In addition, the number of $P a e R 7$ sites within the mammalian genome is underrepresented relative to other hexanucleotides, which would lessen any detrimental effects of heterologous methylation.

In this report, the isolation of four cell lines that express the PaeR7 methylase (A24b1, B6b2, B6c1, and B11b1) is described. No anomalous growth properties were observed for the four strains expressing the PaeR7 methylase gene. These strains were selected based on the strong resistance of their chromosomal DNA and their cotransfected $\phi 80$ DNA to Xho I cleavage. However, completely modified murine adenovirus genomes were not observed even after two rounds of infection through the cell lines expressing the $\mathrm{PaeR7}$ methylase. Interestingly, human adenovirus has been observed to be unmethylated by the host cell's CpG methylase during replication in the nucleus (29). The manner by which adenovirus DNA avoids becoming methylated when replicating is an interesting question. Murine adenovirus is partially methylated at its $\mathrm{PaeR} 7$ sites when grown on methylase-expressing host cells. The reasons for incomplete methylation, even after two passages of growth through methylase-expressing host cells, may stem from lower-thannecessary levels of expression of the methylase or the unavailability of all of the sites during the viral genome replication. However, creation of mammalian cell lines expressing site-specific DNA methylases may be a means of studying the effects of site-specific methylation upon viral gene expression.

The expression plasmids and cell strains reported here may be useful in the study of DNA repair. It has recently been suggested that DNA methylation has a role in DNA repair. Hare and Taylor (30) presented evidence suggesting that 5 -methylcytosine in mammalian cells may assist in strand discrimination for the repair of mismatched bases. Such a mechanism has been determined to operate in E. coli (31-33). Interestingly, 6-methyladenine (resulting from dam modification in vitro) was observed by Hare and Taylor to also be effective in DNA repair.

It also has not escaped our attention that the cell lines expressing high $\mathrm{PaeR} 7$ methylase enzymes are suitable for the introduction and expression of the $P a e R 7$ endonuclease gene. Cells that are expressing a complete bacterial restriction-modification system may in turn be resistant to DNA viral infection

We thank Dr. J. E. Brooks for the gift of purified PaeR7 methylase protein; SIBIA, Inc. for oligonucleotides; Dr. K. R. Spindler for murine adenovirus; Dr. M. F. Law for pMMT341; and Janice Doty for assistance in the preparation of this manuscript. This research was supported by National Science Foundation (PCM84-02963) and SIBIA grants to T.R.G.

1. Gruenbaum, Y., Cedar, H. \& Razin, A. (1982) Nature (London) 295, 620-622.

2. Bird, A. \& Southern, E. M. (1978) J. Mol. Biol. 118, 27-48.

3. Yisraeli, J. \& Szyf, M. (1984) in DNA Methylation: Biochemistry and Biological Significance, eds., Razin, A., Cedar, H. \& Riggs, A. D. (Springer, New York), pp. 353-378.

4. Pollack, Y., Stein, R., Razin, A. \& Cedar, H. (1980) Proc. Natl. Acad. Sci. USA 77, 6463-6467.

5. Wigler, M., Levy, D. \& Perucho, M. (1981) Cell 24, 33-40.

6. Stein, R., Gruenbaum, Y., Pollack, Y., Razin, A. \& Cedar, H. (1982) Proc. Natl. Acad. Sci. USA 79, 61-65.

7. Harland, R. M. (1982) Proc. Natl. Acad. Sci. USA 79, 2323-2327.

8. Gingeras, T. R. \& Brooks, J. E. (1983) Proc. Natl. Acad. Sci. USA 80, 402-406.

9. Cohen, S. N., Chang, A. C. Y. \& Hsu, L. (1972) Proc. Natl. Acad. Sci. USA 69, 2110-2114.

10. Kwoh, T. J., Zipser, D. \& Wigler, M. (1983) J. Mol. Appl. Genet. 2, 191-200.

11. Guerry, P., LeBlanc, D. J. \& Falkow, S. (1973) J. Bacteriol. 116, 1064-1066.

12. Klein, R. D., Selsing, E. \& Wells, R. D. (1980) Plasmid 3, 88-91.

13. Miller, J. (1972) Experiments in Molecular Genetics (Cold Spring Harbor Laboratory, Cold Spring Harbor, NY).

14. Maniatis, T., Fritsch, E. F. \& Sambrook, J. (1982) Molecular Cloning: A Laboratory Manual (Cold Spring Harbor Laboratory, Cold Spring Harbor, NY).

15. Grunstein, M. \& Hogness, D. (1975) Proc. Natl. Acad. Sci. USA 72, 191-195.

16. Theriault, G., Roy, P. H., Howard, R. A., Benner, J. S., Brooks, J. E., Waters, A. F. \& Gingeras, T. R. (1985) Nucleic Acids Res. 13, 8441-8461.

17. Dretzen, G., Bellard, M., Sassone-Corsi, P. \& Chambon, P. (1981) Anal. Biochem. 112, 295-298.

18. Wigler, M., Sweet, R., Sim, G. K., Wold, B., Pellicer, A., Lacy, E., Maniatis, T., Silverstein, S. \& Axel, R. (1979) Cell 16, 777-785.

19. Southern, E. M. (1975) J. Mol. Biol. 98, 503-517.

20. Towbin, H., Staehelin, T. \& Gordon, J. (1979) Proc. Natl. Acad. Sci. USA 76, 4350-4354.

21. Larsen, S. H. \& Nathans, D. (1977) Virology 82, 182-195.

22. Taylor, S. M. \& Jones, P. A. (1982) J. Mol. Biol. 162, 679-692.

23. Adams, R. L. P., Fulton, J. \& Kirk, D. (1982) Biochim. Biophys. Acta 697, 286-294.

24. Jones, P. A. \& Taylor, S. M. (1981) Nucleic Acids Res. 9, 2933-2947.

25. Benedict, W. F., Banerjee, A., Gardner, A. \& Jones, P. A. (1977) Can. Res. 37, 2202-2208.

26. Banerjee, A. \& Benedict, W. F. (1979) Can. Res. 39, 797-799.

27. Brooks, J. E., Blumenthal, R. M. \& Gingeras, T. R. (1983) Nucleic Acids Res. 11, 837-851.

28. Malone, R. E. \& Hoekstra, M. F. (1985) Mol. Cell. Biol. 5, 6110-6118.

29. Doerfler, W., Kruczek, I., Eick, D., Vardimon, L. \& Kron, B. (1982) Cold Spring Harbor Symp. Quant. Biol. 47, 593-603.

30. Hare, J. T. \& Taylor, J. H. (1985) Proc. Natl. Acad. Sci. USA 82, 7350-7354.

31. Pukkila, P. J., Peterson, J., Herman, G., Modrich, P. \& Meselson, M. (1983) Genetics 104, 571-582.

32. Wagner, R. E. \& Meselson, M. (1976) Proc. Natl. Acad. Sci. USA 73, 4135-4139.

33. Glickman, B. \& Radman, M. (1980) Proc. Natl. Acad. Sci. USA 77, 1063-1067. 